\title{
Efficacy in Suppressing Ovulation and Safety of a Low dose Oral Contraceptive in a Continuous Regimen $(84+7)$ With Continuous Ethinyl Estradiol Instead of a Hormone-Free Interval: An Evaluation of Ovulation Suppression and Ovarian Activity
}

\author{
Inna Apolikhina ${ }^{1,2 *}$, Andrey Kuzemin ${ }^{1}$, Victoria Odinokova ${ }^{1}$, Victor Radzinsky ${ }^{3}$, Alexandra Gardner ${ }^{4 *}$ and \\ Gennady Sukhikh ${ }^{1,2}$ \\ ${ }^{1}$ National Medical Research Center of Obstetrics, Gynecology, and Perinatology named after Academician VI Kulakov, Ministry of Health of Russia, Russia \\ ${ }^{2}$ Department of Obstetrics, Gynecology, Perinatology, and Reproductology, Institute of Professional Education, IM Sechenov First Moscow State Medical \\ University, Russia
}

${ }^{3}$ Department of Obstetrics and Gynecology with the course of perinatology of the Peoples' Friendship University of Russia, Russia

${ }^{4}$ Teva LLC, Moscow, Russia

*Corresponding author: Inna Apolikhina, Professor, Obstetrician-Gynecologist, National Medical Research Center of Obstetrics, Gynecology, and Perinatology named after Academician VI Kulakov, Ministry of Health of Russia, Moscow, Russia.

Received Date: January 12, 2020

Published Date: January 29, 2020

\section{Abstract}

Background: This study aimed to evaluate the efficacy of a low dose oral contraceptive in a continuous regimen (84+7) with continuous ethinyl estradiol (EE) instead of a hormone-free interval (HFI) (84 tablets containing 100 mcg levonorgestrel (LNG) + 20 mcg EE, and 7 tablets containing 10 mcg EE; MODELLE $®$ LIBERA, Teva) in suppression of ovulation and ovarian activity.

Methods: A multicenter, open-label, single-treatment, Phase 3 study that evaluated 52 healthy non-pregnant females aged 18 through 35 years, of whom $47(90 \%)$ participants completed the entire 91-day treatment. Evaluation of the efficacy of the formulation in suppressing ovulation was achieved by performing transvaginal ultrasound examinations and determining the serum concentrations of follicle-stimulating hormone (FSH), luteinizing hormone (LH), estradiol, and progesterone.

Results: Overall, $88 \%$ of the participants presented with a lack of ovarian activity during all three 28-day intervals corresponding to a standard cycle. Ovarian suppression was reported in $99 \%$ of all cycles. The serum levels of sex hormones corresponded to the predicted values with allowance for the relevant extended mode of administration of the combined oral contraceptives (COCs). During the post-therapeutic follow-up visit, the restoration of ovulation on the post-therapeutic follow-up visit (the 20th week of the study) was reported in 45 females (87\%, 95\% binomial CI: $74.2 \%, 94.4 \%), 13 \%$ did not return to ovulation.

Conclusions: The efficacy of the study drug in suppressing ovulation was confirmed by the absence of ovarian activity reported in the majority of the participants over the 91-day course of treatment. Ovarian activity was detected in 2 participants (grade 4 according to the Hoogland and Skouby scale, luteinized unruptured follicle), and in each, the relevant activity was observed during the 3rd interval within the 91-day cycle during the 7-day period when only low doses of EE were administered. The underlying basis of the ovarian activity observed during the 7-day EE-only period has not been fully elucidated. The results of the study demonstrate that a low dose oral contraceptive in a continuous regimen (84+7) with continuous EE instead of a hormone-free interval is safe and can be an additional contraceptive option for healthy women seeking decreased menstrual bleeding.

Trial registration: Grls.rosminzdrav.ru (RCT №37 (28.01.2014).

Keywords: Continuous regimen; Oral contraception; Low dose; Hormone-free interval; Modelle ${ }^{\circledR}$ Libera; LoSeasonique

\section{Summary}

Combined oral contraceptives are effective and one of the most popular methods of contraception. Since their appearance, these

medications composition and regimen have undergone changes. The traditional regimens simulate a 28-day menstrual cycle. However, 
91-day prolonged regimens without hormonal free intervals have already been developed. This prolonged regimen allows to decrease the frequency of menstrual-like scheduled bleeding episodes and can meet women's social and cultural reasons.

In this study, the efficacy in suppressing ovulation and safety of low dose oral contraceptive in a continuous regimen without hormonal free intervals were evaluated. The efficacy was evaluated with the help of ultrasound examinations of the ovaries and the serum concentrations of sex hormones measurements: follicle-stimulating hormone, luteinizing hormone, estradiol, and progesterone.

52 healthy non-pregnant women have participated in the study. Suppression of ovarian function and, consequently, the effectiveness of contraceptives was observed in $99 \%$ of all cycles. No pregnancies were detected during the treatment period. The safety profile was also within the expected values. Ovarian function restoration was recorded in $87 \%$ of participants on the 20 th week of the study during the follow-up period.

In conclusion, these results have demonstrated that the low dose combined oral contraceptives in a continuous regimen are effective and safe for healthy women.

\section{Introduction}

COCs are the most used reversible method of birth control and have remained the most popular form of contraception for decades, despite the introduction of other methods of contraception such as parenteral methods [1]. Approximately 9\% of women globally aged 15 to 49 years prefer COCs over other methods of contraception, and this figure reaches $18 \%$ in developed countries [2], while the frequency of use of modern contraceptive methods in developed countries is generally higher. Over the years, the composition of COCs has undergone significant changes: the dose of the estrogen component has been significantly reduced, and new progestogens have been developed and included. The current practice includes administration of multiphase drugs and extended administration regimens with shorter hormone-free intervals (24/4) [1,3-6] in order to increase the efficacy, safety, and acceptability of COCs [711].

Since their initial development over 50 years ago, COCs have been most administered in accordance with the standard regimen that simulates a natural 28-day cycle (21 active pills +7 -day break) [3]. This regimen was developed to mimic natural menstrual cycles. It was not designed out of medical necessity but due to cultural and social practices [12]. COCs represented a revolutionary method of controlling fertility at the time of their creation, which was supposed to be convenient for both women and physicians. The absence of the scheduled bleeding episodes raised doubts regarding the efficacy of the method and could lead to refusals to use this method of birth control, as amenorrhea was clearly associated with the onset of pregnancy some years ago [4]. Today, as there is no longer any doubt regarding the efficacy of COCs, the diagnosis of early pregnancy is relatively easy, and the use of prolonged regimens is a routine practice, the question of the expediency of the administration of COCs in the traditional cyclic regimens remains debatable.

The administration of COCs over prolonged periods of time accompanied by a predictable decrease in the frequency of menstrual-like scheduled bleeding episodes may be desirable for many women. Women express a desire to reduce the frequency of menstruation, including for social and cultural reasons $[5,6,13]$. Reductions in the number of menses per year contribute to improvements in the quality of life by reducing the frequency of menstrual and premenstrual symptoms. The use of prolonged administration regimens leads to a reduction in the incidence of side effects and generally increases the efficacy of contraception [3].

The development of the drugs characterized by continuous administration regimens gained popularity in the early 2000s, while studies on the use of COCs over prolonged periods of time began as far back as the 1970s. In 2003, the FDA approved a new administration regimen for COCs (84/7) [14].

The administration of extended-cycle COCs does not lead to complete suppression of ovarian function. At the beginning of the 7-day hormone-free interval, the activity of the ovaries is minimal [15]. However, due to the lack of active components during the HFI, the activity of the hypothalamic-pituitary-ovarian system axis is slowly restored as the estrogens and progestogens are metabolized. The reduction or modification of the HFI may contribute to an additional decrease in the functional activity of the ovaries [3].

In 2003, the first drug that replaced the HFI with the administration of tablets containing ultra-low doses of EE of 10 $\mathrm{mcg} /$ day was created [3]. The stabilizing period associated with the administration of tablets containing $10 \mathrm{mcg}$ EE instead of placebo suppresses the levels of endogenous estradiol, FSH, and Inhibin-B and leads to increased suppression of ovarian follicular development, reduction in follicular growth and reduction in the risk of ovulation and, accordingly, the risk of unplanned pregnancy [16].

The study drug (MODELLE® LIBERA, Teva LLC Russia; also registered in the USA as LoSeasonique, Teva) is a low dose combined estrogen-progestogen oral contraceptive in a 91-day continuous regimen $(84+7)$ with continuous $10 \mathrm{mcg}$ EE instead of an HFI. The drug suppresses the secretion of gonadotropic hormones. The contraceptive effect is achieved in several ways, the most important of which is through the suppression of ovulation. A single package contains 84 tablets containing 100 mcg LNG and $20 \mathrm{mcg}$ EE each and 7 tablets containing $10 \mathrm{mcg} \mathrm{EE}$ each. Administration of this COC reduces the number of menstrual-like bleeding episodes to just four per year. Over the last 7 days of the prolonged use of the drug (on days 85-91), the administration of 10 mcg of EE instead of placebo is associated with an increase in the suppression of the ovarian follicular apparatus and a decrease in the risk of ovulation. Menstrual-like bleeding after discontinuation 
of the active tablets of the drug is attributed to the absence of the progestin-mediated effect exerted on the endometrium. Meanwhile, residual suppression of the hypothalamic-pituitary-ovarian system and functional activity of the ovaries is retained during this period due to the administration of a small dose of EE [17].

The purpose of this study was to evaluate the efficacy of a low dose oral contraceptive in a continuous regimen $(84+7)$ with continuous EE instead of an HFI in suppressing ovulation and ovarian activity. For this purpose, the following indicators were evaluated:

- $\quad$ Ripening of follicles in the ovaries using transvaginal ultrasound (transvaginal sonography, TVS);

- Determination of serum concentrations of $\mathrm{FSH}, \mathrm{LH}$, estradiol, and progesterone.

Additionally, restoration of ovulation function, the frequency and severity of adverse events and the frequency of pregnancies were evaluated.

\section{Materials and Methods}

\section{Design of the study and population}

The efficacy in suppression of ovulation and safety of a low dose oral contraceptive in a continuous regimen $(84+7)$ was evaluated in an open-label multicenter ( 6 trial sites) study with a single group of female volunteers on the basis of the assessment of the ovarian function and the level of sex hormones (Study DR-101-WH-30007). It was planned to include 60 women in the study with a minimum of 30 women completing the 91-day treatment period. The size of the sample was calculated for the main variable of the study which was the efficacy in suppressing ovulation assessed by the ovarian activity (Hoogland \& Skouby scale). It was assumed that the trial power would be $80 \%(\beta=0.2, z \beta=0.84)$, the significance level $\alpha=0.05(\mathrm{z} \alpha=1.64)$. It was also assumed that the efficacy of the treatment in suppressing ovulation would be established after the drug administration in $75 \%$ of volunteers $(p=0.75)$. The reference value of this indicator was considered equal to $50 \%$ ( $\mathrm{p} 0=0.5$ ). It was also assumed that a difference more than $10 \%(\mathrm{~d}=0.1)$ would indicate clinical importance. Thus, for the final analysis at least 51 female volunteers were required. Considering the possible exclusion of the participants during the treatment period up to $20 \%$, it was necessary to screen at least 60 women for enrollment. One cohort of the participants was analyzed. The baseline characteristics, the frequency of efficacy in suppressing ovulation and safety were assessed and summarized. The significance levels and confidence intervals were calculated as two-sided; the statistical significance of the differences was also two-sided and related to the significance level of 0.05 .

66 healthy females were screened for enrollment into the study. Of these women, 52 sexually active healthy females aged 18 to 35 (with the median age of 26.5 years) who agreed to use COCs as their main method of contraception throughout the entire study period of 91 days and use a double barrier method of contraception (e.g., a condom and spermicide or a diaphragm and spermicide) to prevent pregnancy were included. Of the 14 females who were not enrolled, 8 were not enrolled due to low baseline progesterone levels $(<15.9$ mmol/L), 2 were excluded on the basis of inclusion criteria, 2 were excluded on the basis of exclusion criteria, 1 revoked patient informed consent and 1 was lost to follow-up. Regular spontaneous menstruation occurring approximately once a month or every 2333 days before the screening visit, determining that the subject was ovulating, was among the inclusion criteria. The patients with contraindications to COCs and/or a history of significant adverse events associated with the administration of oral contraceptives were excluded from the study. The exclusion criteria also included the use of injectable hormonal contraceptives over a period of 6 months prior to the screening visit or the presence of a contraceptive implant/hormonal intrauterine device at the time of the screening visit. All participants were instructed during visit 1 a to the study site on the regimen of taking1 tablet once a day at the same time (84 tablets of $100 \mathrm{mcg}$ LNG $+20 \mathrm{mcg}$ EE and 7 tablets of $10 \mathrm{mcg}$ EE) during the 91 days of the treatment period.

The study included 4 stages, during which the patients were obliged to visit their study sites a total of 9 times (visits 1a-9a), corresponding to the relevant weeks of the study (Table 1).

Table 1: Designation of the visits with allowance for the weeks of the study.

\begin{tabular}{|c|c|}
\hline Week & Visit \\
\hline 3 & $1 \mathrm{a}$ \\
\hline 5 & $2 \mathrm{a}$ and 3a \\
\hline 6 & $4 \mathrm{a}$ \\
\hline 10 & $5 \mathrm{a}$ \\
\hline 15 & $6 \mathrm{a}$ \\
\hline 16 & $7 \mathrm{a}$ \\
\hline 17 & $8 \mathrm{a}$ \\
\hline 20 & $9 \mathrm{a}$ \\
\hline
\end{tabular}

\section{Study procedures}

During the screening phase, the participants underwent gynecological examinations, their medical histories were reviewed, and the date of the onset of the last menstruation was determined for each patient. The first TVS examination was also performed during the specified stage to confirm the normal anatomy of both ovaries and to determine the feasibility of their visualization. Prior to the administration of the drug, the levels of sex hormones were determined, and the participants were assessed for their eligibility for participation through the inclusion/exclusion criteria. Visit 1a was organized on day 18-20 of the menstrual cycle. During that stage, the levels of FSH, LH, estradiol, and progesterone were evaluated (Figure 1). The relevant values were recorded as the baseline values. Spontaneous ovulation was assessed by determining the serum level of progesterone. The participants were also subjected to TVS examinations to assess the initial activity of the ovarian follicles. During the subsequent visits, the study drug was administered. The ovarian activity was evaluated using TVS and the Hoogland and Skouby scale [18]; the levels of FSH, LH, estradiol, 
and progesterone were also determined. Throughout the entirety of the study, the participants-maintained diaries recording their intake of medications and any health-related changes. After the completion of the treatment cycle, the restoration of ovulation was evaluated by measuring the serum level of progesterone (Figure 2).

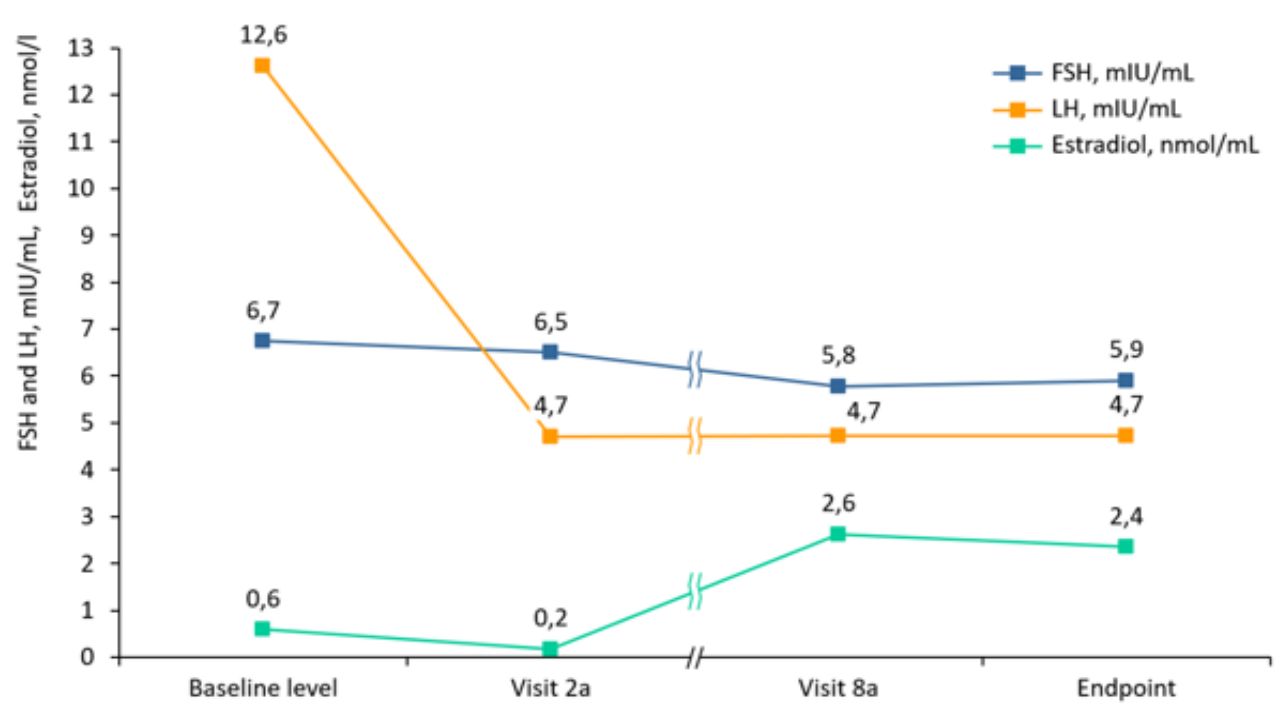

Figure 1: Changes in the FSH, $\mathrm{LH}$, and estradiol levels.

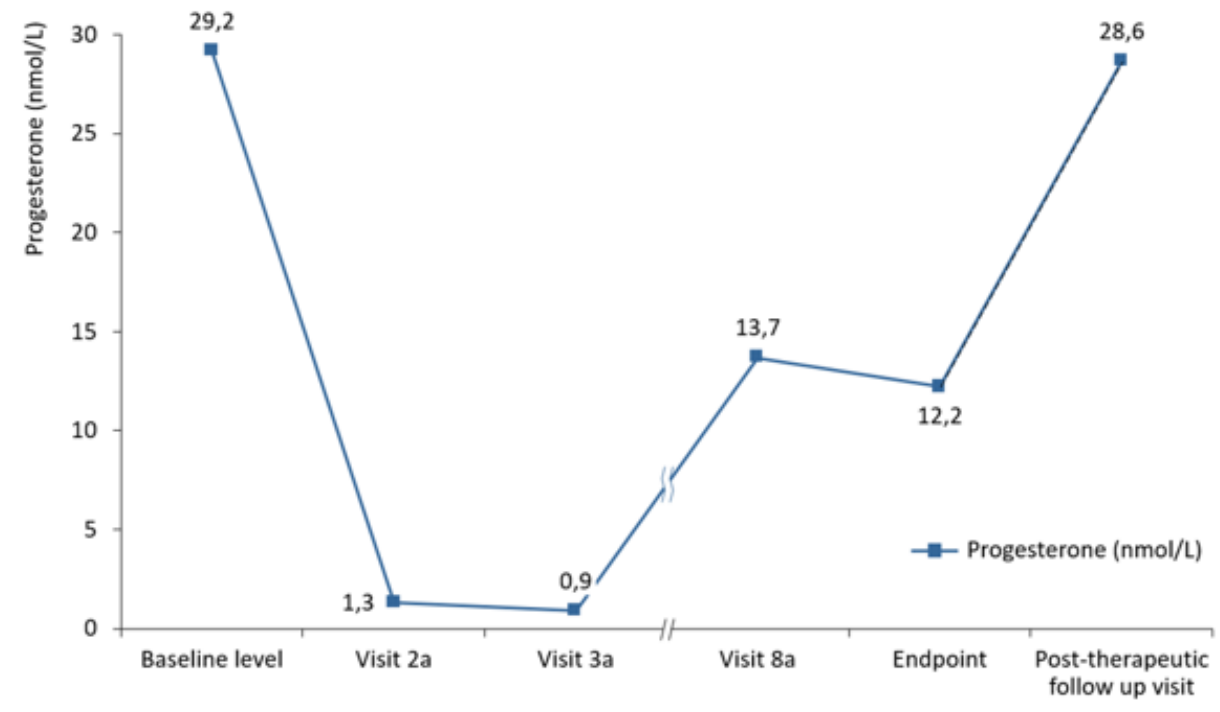

Figure 2: Blood serum level of progesterone.

\section{Evaluation of efficacy in suppressing ovulation}

The ovarian follicular activity evaluation indicators provided by the Hoogland and Skouby scale represented the primary efficacy criteria (percentage of patients with ovarian activity at each TVS examination visit within the active treatment phase). For each participant, a rating from 0 (absence of ovarian activity) to 5 (ovulation with follicle rupture) was determined. Ovarian activity was confirmed at grade 4 (luteinized unruptured follicle) or 5 (ovulation). Furthermore, the suppression of ovulation was evaluated, and the serum contents of the sex hormones (FSH, LH, estradiol, and progesterone) were determined within the framework of the study. During the post-therapeutic follow-up period, the restoration of ovulation was evaluated on the basis of the serum level of progesterone; the relevant tests were repeated until the level of progesterone reached at least $15.9 \mathrm{nmol} / \mathrm{L}$ (but not more than on 6 separate occasions). The incidence of pregnancy was determined on the basis of urinary tests during all visits for all participants throughout the study.

\section{Safety}

The safety analysis was conducted on the basis of the reported adverse events, laboratory test results, vital signs, physical examination data, and information regarding concomitant drug treatment. 
The evaluation of laboratory parameters, including the biochemical analyses, complete blood counts, coagulograms, and determinations of the levels of hormones, were performed by the central laboratory.

The biochemical assay included serum levels of glucose, potassium, calcium, creatinine, urea nitrogen, chloride, total bilirubin, alkaline phosphatase, alanine aminotransferase (ALT), aspartate aminotransferase (AST), total protein, inorganic phosphorus, triglycerides and cholesterol (including high-density lipoproteins (HDL), low-density lipoproteins (LDL), and total cholesterol). The creatinine clearance was calculated using the method developed by Cockcroft-Gault.

\section{Statistical analysis}

Data were processed using SAS®, version 9.1.3. The laboratory data, as well as the data regarding the efficacy in suppressing ovulation and safety, were evaluated using descriptive statistical methods. The patient population receiving treatment (intent-totreat population, ITT) was represented by all the participants receiving treatment who had undergone at least one TVS procedure during the treatment period. The patient population that observed the protocol conditions (modified per protocol population, mPP) was represented by all the participants from the ITT population except for the participants who were excluded from the study for the following reasons:

- $\quad$ Completed less than 82 days of treatment;

- $\quad$ Failed to ingest a total of 3 tablets of study drug consecutively;

- $\quad$ Failed to ingest a total of 9 tablets of study drug over the entire study period;

- $\quad$ Failed to appear for successive TVS procedures during visits 2 a to $4 a$ or visits $5 a$ to $7 \mathrm{a}$.

Summaries of the efficacy data were provided for the ITT and mPP populations. Comparative analysis was not carried out, as the study was conducted in the same group of patients.

Treatment adherence was calculated using the following formula:

Treatment adherence $(\%)=($ actual number of ingested tablets / number of tablets to be ingested*) $\times 100$

Treatment adherence was not calculated for the participants who failed to return the relevant empty blister cards.

All adverse events were encoded using MedDRA, version 17.0.

\section{Results}

\section{Study participants}

The study included 52 healthy females. All participants received at least 1 dose of the study drug and were suitable for evaluation of the safety and efficacy in suppressing ovulation (ITT population); among the specified number of participants, 47 (90\%) of the participants completed the study by participating in all phases of the 91-day treatment period. The majority of the participants were Caucasian (51 participants [98\%], and one participant was of Asian descent [2\%]). The median age was 26.5 years. The study was conducted at 6 study sites located within the Russian Federation.

The treatment of $5(10 \%)$ participants was terminated prematurely, of whom $3(6 \%)$ withdrew because of an adverse event and 2 (4\%) due to protocol violations.

The data regarding the participants enrolled in the study are presented in Tables $2 \& 3$.

Table 2: Baseline characteristics of the patients.

\begin{tabular}{|c|c|}
\hline Parameter & Value \\
\hline Age, years (mean \pm SD) & $26.6 \pm 4.8$ \\
\hline \multicolumn{2}{|l|}{ Race, $\mathrm{n}(\%)$} \\
\hline Caucasians & $51(98)$ \\
\hline Asians & $1(2)$ \\
\hline Body weight, $\mathrm{kg}($ mean $\pm \mathrm{SD})$ & $61.2 \pm 9.8$ \\
\hline Height, cm (mean \pm SD) & $165.4 \pm 7.7$ \\
\hline BMI, kg/m2 (mean \pm SD) & $22.4 \pm 3.4$ \\
\hline \multicolumn{2}{|c|}{ Average volume of menstruum, $\mathrm{n}(\%)$} \\
\hline Scanty & 0 \\
\hline Moderate & $51(98)$ \\
\hline Heavy & $1(2)$ \\
\hline \multicolumn{2}{|c|}{ Duration of bleeding in days, $\mathrm{n}(\%)$} \\
\hline 1 & $0(0)$ \\
\hline 2 & $0(0)$ \\
\hline 3 & $0(0)$ \\
\hline 4 & $8(15)$ \\
\hline 5 & $23(44)$ \\
\hline 6 & $12(23)$ \\
\hline 7 & $9(17)$ \\
\hline 8 & $0(0)$ \\
\hline 9 & $0(0)$ \\
\hline 10 and more & $0(0)$ \\
\hline \multicolumn{2}{|c|}{ History of use of hormonal contraceptives, $n(\%)$} \\
\hline Previous experience & $17(33)$ \\
\hline First experience & $35(67)$ \\
\hline
\end{tabular}

Table 3: The percentage of the participants among the ITT population with ovarian activity according to the Hoogland and Skouby scale at certain point in time during the 91-day period.

\begin{tabular}{|c|c|c|}
\hline $\begin{array}{c}\text { Grade according to } \\
\text { the Hoogland and } \\
\text { Skouby scale }\end{array}$ & Number, $\mathbf{n}(\%)$ & $\begin{array}{c}\mathbf{9 5} \% \text { binomial } \\
\text { confidence intervals }\end{array}$ \\
\hline 0: Lack of activity & $39(75)$ & \\
\hline 1: Potentially active & $11(21)$ & \\
\hline 2: Inactive follicles & $0(0)$ & \\
\hline 3: Active follicles & $0(0)$ & \\
\hline $\begin{array}{c}\text { 4: Luteinized } \\
\text { unruptured follicle }\end{array}$ & $2(4)$ & $0.5 \% ; 13.2 \%$ \\
\hline 5: Ovulation & $0(0)$ & \\
\hline $\begin{array}{c}\text { Participants with } \\
\text { ovarian activity }\end{array}$ & $2(4)$ & \\
\hline
\end{tabular}




\section{Results of efficacy in suppressing ovulation evaluation}

The majority of the participants who took the study drug and returned the empty blister cards in accordance with the protocol followed the treatment regimen instructions precisely (50 participants [96\%]). None of the participants who returned the blister cards miss the pill during the treatment period. One participant (2\%) did not return blister cards and was classified as having failed to follow the instructions of the treatment regimen, the adherence of this participant was not included in the final analysis. Another participant (2\%) ceased to be a participant of the study and was classified as an "absentee".

\section{Evaluation of the state of ovarian follicles using the Hoogland and Skouby scale}

The summary data regarding the proportion of the participants presenting with ovarian activity at a certain point in time during the 91-day treatment period are presented in Table 4. In the ITT population, 39 females (75\%) presented with no ovarian activity at all times during the treatment period. Potential ovarian activity was reported in 11 women (21\%), and 2 participants (4\%; 95\% CI: $0.5 \%, 13.2 \%$ ) presented with confirmed ovarian activity (grade 4 according to the Hoogland and Skouby scale, luteinized unruptured follicle).

The results for the mPP population were comparable: the ovarian activity at a certain point in time during the treatment period was reported in two participants (4\%; 95\% CI: $0.5 \%$, 14.5\%); 11 participants (23\%) presented with potential ovarian activity, and 34 females (72\%) presented with no ovarian activity throughout the treatment period.

The 91-day extended treatment cycle was divided into 3 intervals (i.e., the initial 28 days of the treatment, the subsequent 28 days of the treatment, and the final 35 days of the treatment; these data are presented in Table 4) to determine the number of patientcycles with signs of ovulation. The 91-day period of treatment was divided into such intervals to compare them with the traditional 28day cycle of the oral contraceptive treatment. In the similar studies on the evaluation of the ovarian activity with oral contraceptive treatment in the 21+7 regimen, the 7-day interval was included in the study period. Thereby the duration of the last interval (28 days of taking $100 \mathrm{mcg}$ LNG $+20 \mathrm{mcg}$ EE +7 days of taking $10 \mathrm{mcg}$ EE) was 35 days.

Table 4: The prevalence of the ovarian activity among the ITT population according to the Hoogland and Skouby scale during the 91-day period of treatment.

\begin{tabular}{|c|c|c|}
\hline Grade according to the Hoogland and Skouby scale & Number, $n(\%)$ & $95 \% \mathrm{CI}$ \\
\hline \multicolumn{3}{|c|}{ First interval (the initial 28-day interval of the treatment) } \\
\hline \multicolumn{3}{|l|}{ Number of cycles $n=52$} \\
\hline 0: Lack of activity & $49(94)$ & \\
\hline 1: Potentially active & $3(6)$ & \\
\hline 2: Inactive follicles & $0(0)$ & \\
\hline 3: Active follicles & $0(0)$ & \\
\hline 4: Luteinized unruptured follicle & $0(0)$ & \\
\hline 5: Ovulation & $0(0)$ & \\
\hline Cycles with ovarian activity during the interval & $0(0)$ & $0.0 \%, 6.8 \%$ \\
\hline \multicolumn{3}{|c|}{ Second interval (the subsequent 28-day interval of the treatment) } \\
\hline \multicolumn{3}{|c|}{\begin{tabular}{|l|l} 
Number of cycles $n=47$ & \\
\end{tabular}} \\
\hline 0: Lack of activity & $42(89)$ & \\
\hline 1: Potentially active & $5(11)$ & \\
\hline 2: Inactive follicles & $0(0)$ & \\
\hline 3: Active follicles & $0(0)$ & \\
\hline 4: Luteinized unruptured follicle & $0(0)$ & \\
\hline 5: Ovulation & $0(0)$ & \\
\hline Cycles with ovarian activity during the interval & 0 & $0.0 \%, 7.5 \%$ \\
\hline \multicolumn{3}{|c|}{ Third interval (the final 35-day interval of the treatment) } \\
\hline \multicolumn{3}{|l|}{ Number of cycles $n=47$} \\
\hline 0: Lack of activity & $37(79)$ & \\
\hline 1: Potentially active & $8(17)$ & \\
\hline 2: Inactive follicles & $0(0)$ & \\
\hline 3: Active follicles & $0(0)$ & \\
\hline 4: Luteinized unruptured follicle & $2(4)$ & \\
\hline 5: Ovulation & $0(0)$ & \\
\hline Cycles with ovarian activity during the interval & $2(4)$ & $0.5 \%, 14.5 \%$ \\
\hline Total (91 days) & & \\
\hline
\end{tabular}




\begin{tabular}{|c|c|c|}
\hline \multicolumn{2}{|c|}{ Number of cycles over the therapeutic period $\mathrm{n}=146$} \\
\hline 0: Lack of activity & $128(88)$ & \\
\hline 1: Potentially active & $16(11)$ & \\
\hline 2: Inactive follicles & $0(0)$ & \\
\hline 3: Active follicles & $0(0)$ & \\
\hline 4: Luteinized unruptured follicle & $2(1)$ & \\
\hline 5: Ovulation & $0(0)$ & $0.2 \%, 4.9 \%$ \\
\hline Cycles with ovarian activity during the interval & $2(1)$ & \\
\hline
\end{tabular}

During the $1^{\text {st }}$ interval, the cycles were reported in 52 participants, and during intervals 2 and 3 , the cycles were reported in 47 participants, which was an expected outcome with allowance for the fact that 5 participants left the study prior to completion of interval 2 (day 56). Overall, the lack of ovarian activity was reported in $88 \%$ of the participants during all intervals (91 days) (grade 0 according to the Hoogland and Skouby scale). The number of cycles accompanied by potential ovarian activity (grade 1 according to the Hoogland and Skouby scale) increased during each interval: from 3 cycles (6\%) during interval 1 to $5(11 \%)$ and 8 cycles (17\%) during intervals 2 and 3, respectively. Ovarian activity (grade 4 according to the Hoogland and Skouby scale) was reported in 2 cycles (4\%; binomial CI: $0.5 \%, 14.5 \%$ ) during interval 3 (i.e., during the last 35 days of the 91-day period of treatment).

The analysis of the study results obtained for the patient population that completed the study modified per protocol (mPP) revealed comparable results: ovarian activity (grade 4 according to the Hoogland and Skouby scale) was reported in 2 cycles (4\%; CI: $0.5 \%, 14.5 \%$ ) during interval 3 (i.e., during the final 35 days of the 91-day therapy treatment period). The number of participants with cycles characterized by potential ovarian activity (grade 1 according to the Hoogland and Skouby scale) increased during each successive interval from 3 cycles (6\%) during interval 1 to 5 (11\%) and 8 cycles (17\%) during intervals 2 and 3, respectively. Overall, the majority of the participants (not less than $87 \%$ ) presented with a lack of ovarian activity (grade 0 according to the Hoogland and Skouby scale) over the entire period (91 days).

\section{Evaluation of ovulation suppression}

Over the entire period of the treatment, $96 \%$ of the participants (50 females) from the ITT population presented with ovarian suppression (below grade 4 according to the Hoogland and Skouby scale, with a $95 \%$ binomial CI of $86.8 \%, 99.5 \%$ ). The results for the mPP population were comparable: $96 \%$ of the participants (45 females) presented with ovarian suppression (below grade 4 according to the Hoogland and Skouby scale; 95\% CI: 85.5\%, $99.5 \%)$

Ovarian suppression was reported in $99 \%$ of all cycles during the treatment period. During interval 3, ovarian suppression was reported in all but 2 cycles (45 cycles [96\%; 95\% CI: 85.5\%, 99.5\%]). Analysis of the ovarian suppression observed during all intervals simulating a standard cycle each (i.e., the initial 28 days of the treatment, the subsequent 28 days of the treatment, and the final 35 days of the treatment) demonstrated that all cycles were characterized by ovarian suppression during intervals 1 and 2 (52 cycles [100\%; 95\% CI: 93.2\%, 100\%] and 47 cycles [100\%; 95\% CI: 92.5\%, 100\%], respectively).

\section{Serum levels of sex hormones}

Over the observation period, the mean FSH concentrations decreased from $6.7 \pm 9.4 \mathrm{mIU} / \mathrm{mL}$ to $5.90 \pm 2.59$ at the final examination. The mean LH concentrations decreased more than 2.5-fold from $12.62 \pm 19.59 \mathrm{mIU} / \mathrm{mL}$ (baseline) to $4.730 \pm 2.796$ $\mathrm{mIU} / \mathrm{mL}$ at the final visit. The mean concentrations of estradiol decreased from $0.6 \pm 0.3 \mathrm{nmol} / \mathrm{L}$ (baseline) to $0.17 \pm 0.06 \mathrm{nmol} / \mathrm{L}$ at visit $2 \mathrm{a}$, with an over 4 -fold increase relative to the baseline level to $2.63 \pm 16.09 \mathrm{nmol} / \mathrm{L}$ at visit $8 \mathrm{a}$ and with a mean final value of $2.35 \pm 15.14 \mathrm{nmol} / \mathrm{L}$ at the end of the study.

The mean progesterone concentrations decreased more than 20 -fold from $29.19 \pm 10.29 \mathrm{nmol} / \mathrm{L}$ at baseline to $1.30 \pm 0.82$ $\mathrm{nmol} / \mathrm{L}$ at visit $2 \mathrm{a}$ and $0.93 \pm 0.38 \mathrm{nmol} / \mathrm{L}$ at visit $3 \mathrm{a}$. Despite the increase in progesterone concentrations at visit $8 \mathrm{a}$, they remained approximately 2-fold lower in comparison to the baseline values: $13.68 \pm 81.37 \mathrm{nmol} / \mathrm{L}$ and $12.23 \pm 76.54 \mathrm{nmol} / \mathrm{L}$ at the endpoint visit prior to increasing to approximately the baseline values of $28.64 \pm 15.02 \mathrm{nmol} / \mathrm{L}$ at the post-therapeutic follow-up visit.

\section{Frequency of resumption of ovulation}

At the post-therapeutic follow-up visit, the majority of the participants (45 females [87\%]; 95\% CI: 74.2\%, 94.4\%) presented with resumption of ovulation (i.e., the progesterone concentrations reached or exceeded $15.9 \mathrm{nmol} / \mathrm{L}$ ), 13\% did not return to ovulation. The concentrations of progesterone at visit 9a were below $15.9 \mathrm{nmol} / \mathrm{L}$ in 23 participants (44\%). It was expected that the progesterone levels in these participants would be additionally measured up to 5 times after visit 9 a in accordance with the protocol (i.e., during 6 visits in total, visits 9a-f) until the relevant values would reach or exceed $15.9 \mathrm{nmol} / \mathrm{L}$. Among these 23 participants, 16 patients returned for additional visits, and the relevant progesterone concentrations reached or exceeded 15.9 nmol/L. Five females either did not return for additional visits or returned for fewer than 5 additional visits, and the relevant progesterone concentrations did not reach or exceed $15.9 \mathrm{nmol} / \mathrm{L}$, while the progesterone concentrations in 2 participants remained below $15.9 \mathrm{nmol} / \mathrm{L}$ after 5 additional visits (after 6 measurements in total). Since the primary aim of the study was to assess the suppression of ovulation, not the resumption of it, additional data indicating the return to ovulation were not documented. 


\section{Incidence of pregnancy}

No pregnancy cases were reported among the participants during the study.

\section{Safety evaluation results}

Incidence of adverse events (AE): The majority of the participants (45 females [87\%]) reported at least 1 adverse event. No lethal outcomes were reported during the study. Two participants (4\%) reported at least 1 adverse event with severe intensity, and 3 participants (6\%) experienced adverse events that led to the termination of their participation in the study. One event was recognized as a serious adverse event, and two events were found to be related to the treatment. Expect for the related irregular bleeding (metrorrhagia) reported by the majority of the participants (31 females [60\%], Table 5), all treatment-related adverse events were reported in no more than 2 participants (4\%). One subject with the registered metrorrhagia was a 22-year old female who experienced this adverse event starting on day 11 that was considered resolved on day 42 . As a result of the event, study treatment was withdrawn, and the subject discontinued from the study on day 58. Expect for 1 adverse event with severe intensity in the form of an unrelated toothache reported in 1 participant (2\%), all adverse events were mild (28 participants [54\%]) or moderate (16 participants [31\%]) in severity.

Table 5: All adverse events associated with the treatment.

\begin{tabular}{|c|c|}
\hline $\begin{array}{c}\text { Organ system class } \\
\text { Participants with at least 1 AE associated with } \\
\text { treatment }\end{array}$ & Number, $\mathbf{n}(\mathbf{\%})$ \\
\hline $\begin{array}{c}\text { Disorders of the reproductive system and } \\
\text { mammary glands }\end{array}$ & $31(65)$ \\
\hline Metrorrhagia & $31(60)$ \\
\hline Ovarian cyst & $1(2)$ \\
\hline Disorders of the gastrointestinal tract & $2(4)$ \\
\hline Nausea & $2(4)$ \\
\hline Mental disorders & $2(4)$ \\
\hline Anxiety & $1(2)$ \\
\hline Tearfulness & $1(2)$ \\
\hline Disorders of the skin and subcutaneous tissue & $1(2)$ \\
\hline Drug-induced rash & $1(2)$ \\
\hline
\end{tabular}

Two participants discontinued the treatment because of the protocol violation. One subject was a 20-year old female who experienced a serious adverse event (hospitalization with the suspected appendicitis) of nontreatment-related moderate ovulation pain starting on day 24 that was considered resolved on day 27. As a result of the event, the treatment was withdrawn, and the subject was discontinued from the study on day 41. Another participant who discontinued from the study on day 13 was a 35-year old female. The subject experienced an adverse event of moderate study drug induced dermatitis starting on day 9. The event was considered as resolved on day 18.

No clinically significant changes in the clinical laboratory indicators, blood serum biochemical indicators, complete blood count indicators, blood clotting indicators or other changes in vital signs were observed. None of the results of the clinical laboratory studies and physical examination data collected during the observation period demonstrated clinically significant abnormalities.

\section{Discussion}

This multicenter, open-label, non-randomized study involving one group of patients was conducted to evaluate the efficacy in suppression of ovulation and safety of a low dose oral contraceptive in a continuous regim en $(84+7)$ on the basis of evaluation of the ovarian function and sex hormone levels in females aged 18-35 in the Russian Federation.

Previous studies have demonstrated that a COC regimen that entails administration of $10 \mathrm{mcg}$ LNG and $20 \mathrm{mcg}$ EE over a period of 84 days followed by subsequent administration of 10 mcg EE over 7 additional days is safe and effective throughout the 91-day treatment period [15].

When used in accordance with the recommendations, a 91day regimen of a low dose oral contraceptive is over 99\% effective at pregnancy prevention and demonstrates a Pearl index value of 2.74 (including typical use in females failing to observe the relevant recommendations). Among compliant subjects $18-35$ years old, the Pearl Index was 1.73 [15].

Although the Pearl index was not determined within the framework of the present study, the efficacy of the MODELLE® LIBERA preparation in suppressing ovulation was clearly demonstrated by the fact that $75 \%$ of the participants presented with a lack of ovarian activity over the 91-day period of treatment, as demonstrated by the analysis of the results of TVS and the Hoogland and Skouby scale scores. Potential ovarian activity was observed in approximately $1 / 5$ of the patients (11 participants [21\%]), although no active follicles were observed in any of the participants. Analysis of the ovarian activity observed during 3 intervals simulating a standard cycle each and lasting from 28 to 35 days (the score according to the Hoogland and Skouby scale) demonstrated a slight increase in the ovarian activity over time, manifesting the fact that the number of potentially active cycles (grade 1 according to the Hoogland and Skouby scale) increased with each subsequent interval, from 3 cycles $(6 \%)$ during interval 1 to $5(11 \%)$ and 8 cycles (17\%) during intervals 2 and 3, respectively.

The sustainability of the efficacy of the MODELLE® LIBERA preparation in suppression of ovulation is further demonstrated by the fact that the results collected within the framework of this study were comparable for the ITT and MPP populations.

Ovarian activity was reported in 2 participants (grade 4 according to the Hoogland and Skouby scale, luteinized unruptured follicle), and in both cases, this activity was observed during interval 3 of the 91-day cycle, over the 7-day period when $10 \mathrm{mcg}$ EE doses were administered. The cause of the ovarian activity in these 2 patients during the 7-day period when low EE doses were administered has not been determined. It was reported within the framework of a study conducted by Baerwald and Pierson that the 
onset of ovarian activity and the stability of ovarian suppression fluctuated over several cycles of COCs. The growth of follicles was also demonstrated to be highest during the hormone-free interval [17].

Overall, the changes in sex hormone levels in the ITT population in response to the 91-day treatment with the study drug were expected for prolonged COC usage [19]. Prolonged suppression of serum levels of FSH, estradiol, and LH were also indicative of ovarian suppression. The return to ovulation was documented on the posttherapeutic visit (on the 20th week) in $87 \%$ of women; $13 \%$ did not return to ovulation. The latter is likely attributable to the fact that certain participants did not return for follow-up measurements of progesterone concentrations according to the protocol.

Overall, the safety results obtained within the framework of this study convincingly demonstrated that MODELLE® LIBERA can safely be administered for contraceptive purpose to healthy females. The frequency and the profile of the reported adverse events corresponded to the predicted values for the study drug and the specified treatment regimen. The AEs associated with the treatment were mostly mild in severity, and the frequency of their occurrence aligned with the expectations. The study limitations include cases of metrorrhagia and ovarian cyst, since the study of these issues was not the aim of the present trial. Overall, none of the potentially clinically significant changes in the results of clinical laboratory studies (serum biochemical assay, complete blood count, and blood coagulation analysis), vital signs (heart rate, diastolic blood pressure and systolic blood pressure), body weight or physical examination results (including body weight) were attributed to the treatment with the study drug.

\section{Conclusion}

The results of the study demonstrate that a low dose oral contraceptive in a continuous extended regimen (84+7) with continuous EE instead of an HFI is safe in accordance with the prescribed regimen. The study drug can be an additional extended cycle COC option that offers effective suppression of ovulation and pregnancy prevention in healthy females while decreasing the number of menstruations to only four per year. The results obtained correspond to the data of previous clinical studies of low dose oral contraceptives in 91-day regimens (84 tablets of $100 \mathrm{mcg}$ LNG +20 mcg EE and 7 tablets of $10 \mathrm{mcg}$ EE).

\section{Declarations}

\section{Ethics approval and consent to participate}

The study was approved by Council on Ethics of Russian Ministry of Health, and the local ethics committees of all participating sites and conducted in accordance with the protocol and amendments thereto of the ethical principles of the Helsinki Declaration of the World Medical Association (Seoul, 2008), ICH GCP, and in compliance with the relevant regulatory documents and legislation of the Russian Federation. All participants were required to provide informed consent.

\section{Consent for publication}

Not applicable.

\section{Availability of data and material}

The datasets generated and analysed during the current study available from the corresponding author on reasonable request.

\section{Funding}

The study was funded by Teva LLC Russia.

\section{Acknowledgement}

The authors acknowledge Yakov Pakhomov and Irina Bode of Medical Adviser's Group, part of CRA-club LLC, for their assistance and provision of medical writing support in the development of this manuscript. Medical writing support was funded by Teva LLC, Moscow, Russia.

The authors acknowledge all the investigators who took part in this project: Petrova V, Parsadanyan S, Gurskaya T, Manko M, Chernyshova L, Razmakhnina N, Shpachenko Ya, Baranova N, Rybak O, Guseva E, Kovalev V, Kudryavtseva E, Islamidi D, Majorchik E, Khasanov A, Abdrakhmanova A, Tagirova L, Garifullova Yu, Grigoryan F, Plotnikov A, Osmanova S, and Kabilova L.

\section{Competing Interests}

Inna Apolikhina, Andrey Kuzemin, Victoria Odinokova, Viktor Radzinsky, and Gennady Sukhih have participated as investigators in studies conducted by Teva LLC Russia. Viktor Radzinsky is a member of the Teva advisory board. Alexandra Gardner is a fulltime employee of Teva LLC Russia.

\section{Authors' Contributions}

All authors contributed to the data interpretation, reviewed drafts of the manuscript and approved the final draft for submission.

\section{Conflict of Interest}

Authors declare no conflict of interest.

\section{References}

1. Jones J, Mosher W, Daniels K (2012) Current contraceptive use in the United States, 2006-2010, and changes in patterns of use since 1995. Natl. Health Stat. Report 60: 1-25.

2. (2011) The United Nations | World Contraceptive Use 2011.

3. Benson LS, Micks EA (2015) Why Stop Now? Extended and Continuous Regimens of Combined Hormonal Contraceptive Methods. Obstet Gynecol Clin North Am 42(4): 669-681.

4. Thomas SL, Ellertson C (2000) Nuisance or natural and healthy: should monthly menstruation be optional for women? Lancet 355(9207): 922924.

5. Edelman A, Lew R, Cwiak C, Nichols M, Jensen J (2007) Acceptability of contraceptive-induced amenorrhea in a racially diverse group of US women. Contraception 75(6): 450-453.

6. Côté I, Jacobs P, Cumming D (2002) Work loss associated with increased menstrual loss in the United States. Obstet Gynecol 100(4): 683-687.

7. Burkman R, Bell C, Serfaty D (2011) The evolution of combined oral contraception: improving the risk-to-benefit ratio. Contraception $84(1)$ : 19-34. 
8. Edelman AB, Gallo MF, Jensen JT, Nichols MD, Grimes DA, et al. (2005) Continuous or extended cycle vs. cyclic use of combined hormonal contraceptives for contraception. Cochrane Database Syst Rev 3: CD004695.

9. Lawrie TA, Helmerhorst FM, Maitra NK, Kulier R, Bloemenkamp K, Gülmezoglu AM (2011) Types of progestogens in combined oral contraception: effectiveness and side-effects. Cochrane Database Syst Rev 5: CD004861.

10. Bitzer J, Simon JA (2011) Current issues and available options in combined hormonal contraception. Contraception 84(4): 342-356.

11. Read CM (2010) New regimens with combined oral contraceptive pills moving away from traditional 21/7 cycles. Eur J Contracept Reprod Heal Care 15: S32-S41.

12. Kimble T, Thurman A, Schwartz J (2011) Currently available combined oral contraception. Expert Rev Obstet Gynecol 6(5):525-538.

13. Schwartz JL, Creinin MD, Pymar HC (1999) The trimonthly combination oral contraceptive regimen: is it cost effective? Contraception 60(5): 263-267.
14. (2003) Seasonale (Levonorgestrel/Ethinyl Estradiol Tablets). Current US Prescribing Information.

15. Kroll R, Reape KZ, Margolis M (2010) The efficacy and safety of a lowdose, 91-day, extended-regimen oral contraceptive with continuous ethinyl estradiol. Contraception 81(1): 41-48.

16. Reape KZ, DiLiberti CE, Hendy CH, Volpe EJ (2008) Effects on serum hormone levels of low-dose estrogen in place of placebo during the hormone-free interval of an oral contraceptive. Contraception $77(1)$ : 34-39.

17. Baerwald AR, Pierson RA (2004) Ovarian follicular development during the use of oral contraception: a review. J Obstet Gynaecol Can 26(1): 1924.

18. Hoogland HJ, Skouby SO (1993) Ultrasound evaluation of ovarian activity under oral contraceptives. Contraception 47(6): 583-590.

19. (2019) LoSeasonique: Uses, Dosage \& Side Effects - Drugs.com. 